\title{
The Gravitational Wave Detector "Mario Schenberg”: Status of the Project
}

\author{
O D Aguiar ${ }^{1}$, L A Andrade ${ }^{1}$, L Camargo Filho ${ }^{4}$, C A Costa ${ }^{1}$, \\ J C N de Araújo ${ }^{1}$, E C de Rey Neto ${ }^{1}$, S T de Souza ${ }^{2}$, A C Fauth ${ }^{4}$, \\ C Frajuca $^{5}$, G Frossati ${ }^{7}$, S R Furtado ${ }^{1}$, V G S Furtado ${ }^{1}$, \\ N S Magalhães ${ }^{6}$, R M Marinho Jr. ${ }^{3}$, E S Matos ${ }^{1}$, M T Meliani ${ }^{1}$, \\ J L Melo ${ }^{1}$, O D Miranda ${ }^{1}$, N F. Oliveira Jr. ${ }^{2}$, K L Ribeiro ${ }^{1}$, \\ K B M. Salles ${ }^{1}$, C Stellati ${ }^{3}$, W F Velloso Jr. ${ }^{1}$
}

\author{
1. Instituto Nacional de Pesquisas Espaciais - Divisão de Astrofísica, São José dos Campos, SP, Brazil \\ 2. Universidade de São Paulo, Instituto de Física, São Paulo, SP, Brazil \\ 3. Instituto Tecnológico de Aeronáutica, São José dos Campos, SP, Brazil \\ 4. Universidade Estadual de Campinas, Instituto de Física, Campinas, SP, Brazil \\ 5. Centro Federal de Ensino Tecnológico de São Paulo, São Paulo, SP, Brazil \\ 6. Universidade Bandeirante de São Paulo, São Paulo, SP, Brazil \\ 7. Leiden University, Kammerlingh Onnes Laboratory, Leiden, The Netherlands
}

\begin{abstract}
The first phase of the Brazilian Graviton Project is the construction and operation of the gravitational wave detector Mario Schenberg at the Physics Institute of the University of São Paulo. This gravitational wave spherical antenna is planned to feature a sensitivity better than $\mathrm{h}=10^{-21} \mathrm{~Hz}^{-1 / 2}$ at the $3.0-3.4 \mathrm{kHz}$ bandwidth, and to work not only as a detector, but also as a testbed for the development of new technologies. Here we present the status of this detector.
\end{abstract}

\section{The Project ${ }^{1}$}

The antenna will weight about $1150 \mathrm{~kg},{ }^{2}$ which is only a factor of 2 less massive than existent antennas. By cooling it to much lower temperatures (15-20 mK), however, and by making it spherical (omnidirectional), we expect a considerable improvement in sensitivity. ${ }^{3}$

The project will be divided in two phases:

\section{GRANTED phase and detector phase}

In the Gravitational Radiation ANtenna TEchonology Demonstration phase (GRANTED), we will test the following aspects:

1) the feasibility to construct a heavy $(\sim 1150 \mathrm{~kg})$ copper-aluminum $(94 \%-6 \%)$ spherical mass, which presents a high $\left(\mathrm{Q}_{\mathrm{m}}>10^{7}\right)$ mechanical Figure of Merit at $20 \mathrm{mK} ;^{4}$

2) the feasibility to quickly (less than a week) cool such a mass down to $20 \mathrm{mK} ;^{5}$

3) the feasibility to extract and analyze data from a transducer attached to the spherical surface;

4) the feasibility to protect such a spherical mass from laboratory and environment vibrations by the use of designed vibration isolation systems for:

$$
\begin{aligned}
& \text { - the mass suspension, } \\
& \text { - the dilution refrigerator, } \\
& \text { - the transducer wiring. }
\end{aligned}
$$

\section{DETECTOR phase}

After completing the technology demonstration phase (GRANTED), we intend to construct and place a set of high sensitivity transducers attached to the surface of the sphere, transforming the above prototype into an effective gravitational wave detector, the Mario SCHENBERG detector, in the 3.0-3.4 kHz frequency bandwidth. 


\section{The Scientific Goals of the Present Detector}

A $65 \mathrm{~cm}$-diameter $\mathrm{Cu}-\mathrm{Al}(94 \%-6 \%)$ antenna will be covering the $3.0-3.4 \mathrm{kHz}$ bandwidth, which has astrophysical importance. It would be operating in coincidence with the Dutch Mini-GRAIL and the Italian SFERA antennas, and some large laser interferometer detectors, which can also cover such high frequencies with similar sensitivities. ${ }^{6}$

The astrophysical events that we anticipate as probable candidates for detection in the 3.0-3.4 $\mathrm{kHz}$ region, where the SCHENBERG detector will be sensitive, are: ${ }^{7}$

- $\quad$ core colapse in supernova events;

- $\quad$ neutron stars going to hydrodynamical instability;

- $\quad$ quakes and oscillations of neutron stars (f modes) induced by the falling of matter in binary systems;

excitation of the first quadrupole normal mode of $\sim 3.8$ solar-mass black holes; coalescence of $\sim 1.9$ solar-mass neutron stars and/or black holes.

We also can speculate some "exotic" sources such as:

- rotation of bosonic or strange matter stars at $\sim 1.6 \mathrm{kHz}$;

- inspiralling of mini-black hole binaries.

\section{The Schenberg group}

The group is divided in six major subjects:

1) Cryogenics with Prof. Nei Oliveira Jr. (Co-PI), Prof. Giorgio Frossati (Co-PI), Karla Salles (Master student), Sérgio Furtado (Master student), and Sérgio de Souza (Master student),

2) Astrophysical Sources and Gravitation with Dr. José Carlos de Araújo, Dr. Oswaldo Miranda, Dr. Mara Meliani, and Dr. Edgard de Rey Neto, ${ }^{8}$

3) Vibration Isolation with Dr. Walter Velloso Jr. and José Melo (Ph.D. student), ${ }^{9}$

4) Transducers with Dr. Carlos Frajuca, Kilder Ribeiro (Ph.D. student), and Luiz Andrade (Ph.D. student),

5) Cosmic Ray Detection Hardware with Dr. Anderson Fauth and Lúcio Camargo Filho (undergraduate student),

6) Data Analysis with Dr. Rubens Marinho Jr., Dr. Nadja Magalhães, Claudemir Stellati (Ph.D. student), César Costa (Master student), Valéria Furtado (Master student), and Emílio Matos (Master Student). ${ }^{10}$

The Brazilian group is developing this project in collaboration with the Dutch group and all four major groups operating cryogenic resonant mass antennas (ALLEGRO, NAUTILUS \& EXPLORER, AURIGA and NIOBÈ). It also receives collaboration from researchers and professors working at some other Brazilian institutions and universities and at some laser interferometer projects (LIGO, VIRGO, and LISA). 


\section{The Status of the Detector}

\subsection{Site preparation}

The site preparation at the University of São Paulo is under the coordination of N. Oliveira Jr., and is almost completed. A pit has been constructed in one of his laboratory rooms, where the detector will be located. There is one hydraulic piston inside a concrete column at each of the four corners of this pit. These pistons form a hydraulic system that can lift up to 2 meters a reinforced 4-ton concrete base, on which the detector will be assembled. A crane has been installed on the top of a sliding structure connected to the upper part of the room walls. This crane together with the hydraulic system will provide some flexibility for the detector assembly.

\subsection{Cryogenic chambers}

The cryogenic chambers have been designed by G. Frossati and collaborators. They will be shipped from KADEL (USA) in October of 2001, and will be assembled on the concrete base as soon as they arrive at the University of São Paulo.

Frossati has also designed the dilution refrigerator that is going to be used in the detector.

\subsection{Vibration isolation system}

The vibration isolation system that will be used in the Mario Schenberg detector is the product of a technology developed by various gravitational wave detector groups in a few decades of experience. The designed one uses $\mathrm{C}$ springs that were proposed by Italians collaborators. Melo and Velloso have done some detailed calculations using this design and a finite element software (NASTRAN). They were able to design a vibration isolation system free from resonances close to the $3.0-3.4 \mathrm{kHz}$ frequency band. This system, that will be inside the cryogenic chambers, together with a room temperature vibration isolation system very similar to the one used by the ALLEGRO detector, will be able to attenuate more than 360 $\mathrm{dB}$ the vibrations coming though the antenna suspension system.

This system will be cast at ITALBRONZE (Brazil), and will be delivered by the end of 2001 .

\subsection{Sphere}

A first spherical antenna has been cast by ITALBRONZE (Brazil) at the beginning of 2001. It was sent to the Dutch group for testing.

The Schenberg antenna will be cast at ITALBRONZE by the end of 2001.

\subsection{Transducers}

We plan to use a set of seven two-mechanical-mode parametric transducers pumped at about $10 \mathrm{GHz}$ for monitoring the first monopole (one transducer) and first five quadrupole (six transducers) modes of the spherical antenna. This will be done in collaboration with the Australian group and with other groups in Brazil.

\subsection{Schedule}

The first cryogenic run is schedule to the beginning of 2002 and the first transducer test to about six months later. Finally, we plan to put the detector in a commissioning phase sometime in 2003. 


\section{Acknowledgments}

This work has been supported by FAPESP (under grants No. 1998/13468-9, 1996/01872-4, 1997/06024-4, 1999/10809-2, 2000/10374-5, 2001/04189-3, 2001/04086-0, 2001/04226-6), CNPq (under grants No. 300619/92-8 and AVG/450994/01-5), CAPES and INPE/MCT.

\section{References}

\footnotetext{
${ }^{1}$ Proposal submitted in November of 1998 and approved by FAPESP in April 2000.

${ }^{2}$ The initial proposal was a $\mathrm{CuAl}(6 \%) 800 \mathrm{~kg}$ buckyball antenna. However, in common agreement with the Mini-GRAIL and SFERA groups we decided to change it to a $\mathrm{CuAl}(6 \%) 1150 \mathrm{~kg}$ spherical antenna. The high frequency operation of the antenna requires that the transducers be clamped at the antenna surface instead of bolted, therefore there would be no need for flat surfaces (normally required for bolting transducers) and so, for a polyhedron shape antenna. The natural spherical shape also has more mass (more cross section to the waves). The diameter was increased too (from $0.6 \mathrm{~m}$ to $65 \mathrm{~cm}$ ) because we had more room in the cryogenic chambers and because the $3.0-3.4 \mathrm{kHz}$ frequency window seemed more interesting from the astrophysical point of view of the gravitational wave sources listed on section 2 compared to the original $3.9-4.3 \mathrm{kHz}$ frequency one. For example, for hydrodynamical instabilities of neutrons stars, Houser (1998 Mon. Not. R. Astron. Soc. 299, 1069) showed that a frequency band below $3.5 \mathrm{kHz}$ would have more chances to see a stronger signal.

${ }^{3}$ Forward, R L 1971 General Relativity and Gravitation 2 149. Wagoner, R V and Paik, H-J. 1977, Proceedings of the Academia Nazionale dei Lincei, International Symposium on Experimental Gravitation, Pavia, Italy, Sept. 1976, p.257. Johnson, W W and Merkowitz, S M 1993 Physical Review Letters 702367 . Coccia, E. 1997, $14^{\text {th }}$ International Conference on General Relativity and Gravitational, Florence, 1995, World Scientific, Singapore, pp. 103. Waard, A and Frossati, G 2000, Proceedings of the Third Edoardo Amaldi Conference on Gravitational Waves, 1999, Pasadena, California. AIP Conference Proceedings. American Institute of Physics 523, 268.

${ }^{4}$ Frossati, G et all. 1997, Proceeding of the First International Workshop of Omnidirectional Gravitational Radiation Observatory, São José dos Campos, Brazil, May 26-31, 1996, edited by W F Velloso, O D Aguiar, and N S Magalhães, World Scientific, Singapore, 179.

${ }^{5}$ This has already been demonstrated by the Dutch group (www.minigrail.nl) in 2001.

${ }^{6}$ Proceedings of the Third Edoardo Amaldi Conference on Gravitational Waves, 1999, Pasadena, California. AIP Conference Proceedings. American Institute of Physics 523 (2000)

${ }^{7}$ Wagoner, R V 1984 Ap.J. 278 345. Schutz, B F 1997, Relativistic Astrophysics and Gravitational Radiation, eds. J-A Marck and J-P Lasota, Cambridge Univ. Press. Anderson, N and Kokkotas, K D 1996 Phys. Rev. Lett, 77 4134. Anderson, N; Araújo, M E; and Schutz, B F 1993 Class. Quantum Grav. 10 757. Friedman, J L; Ipser, J R; and Parker, L 1989 Phys. Rev. Lett. 623015. Haensel, P; Lasota, J P; and Zdunik, J L astro-ph/9905036, 5 May 1999. Nakamura, T; Sasaki, M; Tanaka, T; and Thorne, K S 1997 Ap. J. 487 L139. Thorne, K S 1987, 300 Years of Gravitation, edited by S. Hawking and W. Israel, Cambridge Univ. Press, Cambridge, pp. 330. Harry, G M; Stevenson, T R; and Paik, H-J 1996 Phys. Rev. D54 2409.

${ }^{8}$ De Araújo, J C N; Miranda, O D; and Aguiar, O D 2001 Ap. J. 550 368. Meliani. M T; De Araújo, J C N; and Aguiar, O D 2000 Astron. Astrophys. 358 417. De Araújo, J C N; Miranda, O D; and Aguiar, O D 2000 Phys. Rev. D61 124015. Novello, M; Lorenci, V A; Freitas, L; and Aguiar, O D 1999 Phys. Lett. A 254 245. Mosquera Cuesta, H J; De Araújo, J C N; Aguiar, O D; and Horvath, J E 1998 Phys. Rev. Lett. 802988.

${ }^{9}$ Velloso Jr., W F; Melo, J L; and Aguiar, O D 2000 Rev. Sci. Instrum. 712552.

${ }^{10}$ Magalhães, N S; Aguiar, O D; Frajuca, C; and Marinho Jr., R M 2001 Nucl. Instrum. Meth. Phys. Res. A457 175. Magalhães, N S; Aguiar, O D; Frajuca, C; Marinho Jr., R M; and Chiang, J 2001 Nucl. Instrum. Meth. Phys. Res. (in press). Marinho Jr., R M; Magalhães, N S; Aguiar, O D; and Frajuca, C 2001 Phys. Rev. D (in press). Magalhães, N S; Johnson, W W; Frajuca, C; and Aguiar, O D 1997 Ap. J. 475 462. Magalhães, N S; Aguiar, O D; and Frajuca, C 2001 Phys. Rev. D (in press). Magalhães, N S; Aguiar, O D; Frajuca, C 1997 Gen. Rel. Grav. 29, 1511. Magalhães, N S; Johnson, W W; Frajuca, C; and Aguiar, O D 1995 Mon. Not. R. Astr. Soc 274670.
} 